Explanations in criminology

Behaviour and Misbehaviour: Explanations and Non-Explanations. By Nigel Walker. Pp. ix +154 (Blackwell: Oxford, 1977.) $£ 5$.

Most new disciplines go through a period of introspection in which they examine the philosophical status of their theories and the adequacy of their methods. Criminology has not yet emerged from this stage, and in Behaviour and Misbehaviour Professor Walker examines the nature of explanation in that subject.

$\mathrm{He}$ distinguishes between "necessity explanations" and "possibility explanations". The former are used to explain regular events such as the daily rising of the Sun or the expansion of a gas on heating, the latter to explain exceptional or unique events. If an iceberg appears as far south as Rockall, we do not ask why it was necessarily there but what made it possible for it to be there: the answer required is a narrative involving such factors as $a$ particularly hard winter in Greenland, the prevailing ocean currents, and the existence of strong north-westerly winds. Professor Walker calls the former type of explanation "scientific" and the latter "narrative". $\mathrm{He}$ then argues that because much criminal behaviour is exceptional, criminologists may have to be satisfied with narrative rather than scientific explanations. $\mathrm{He}$ is probably right: even rates of crime, as well as individual crimes, are determined by many interacting features some of which may be unique to a given society. But it is not clear that the difference between theories in the physical and social sciences is entirely captured by the distinction between "narrative" and "scientific" explanations: as Professor Walker acknowledges, even the physical scientist usually has to fall back on the narrative explanation to account for individual events such as the failure of a bridge.

A further distinction drawn by Professor Walker is that between explanation by analogy and explanation in terms of mechanisms. The difference is by no means clear-cut: an explanation by analogy may in fact be implicitly proposing a mechanism the details of which are not fully worked out. When Harvey had the insight that the heart was a pump was he suggesting an analogy or a mechanism? Exception can be taken to Walker's contention that explaining human behaviour by computer simulation is only giving an analogy: a computer program can rep- resent the mechanisms underlying behaviour in just the same way as a set of equations can represent the mechanisms underlying the behaviour of a physical system.

Some of Professor Walker's omissions are suprising: in discussing scientific explanations he says little about formal rigour, simplicity, elegance or generality. He does, however, rightly castigate his fellow criminologists for the idiocy of some of their disputations such as the controversy over whether a given phenomenon can only have a single explanation and the dispute about the range of phenomena that fall within the province of criminology. He also gives short shrift to the arguments

\section{Enzyme kinetics}

Enzyme Kinetics. By D. V. Roberts. Pp. 326. (Cambridge University : Cambridge, London, New York and Melbourne, 1977.) Hardback £15; paperback $£ 5.50$.

THIS book deals with the use of mathematical expressions to describe the behaviour of enzymes and enzyme systems. Although the author works through the derivations of many of the equations that he presents, some familiarity with the use of determinants and the calculus is necessary if the reader is to follow the arguments used, and the book could not be recommend to mathematically weak students. For those who are not frightened-off by the mathematical treatments used, the author's comparison between the use of determinants and the schematic King-Altman procedure for deriving initial-rate equations is valuable, as is his treatment of the use of the LaplaceCarson operator method for solving the differential equations describing presteady-state behaviour. At times the derivation of equations seems to take precedence over consideration of the phenomena that they attempt to describe. In the chapter on allosteric proteins, for example, it is unfortunate that the author does not give more emphasis to the predictions made by the different models that he considers and to ways in which they may be distinguished.

The coverage given to different aspects of enzyme kinetics varies greatly and some topics, such as the effects of $p \mathrm{H}$ and temperature on enzyme activity, are given rather shallow treatments. In some places the of Peters, Winch and others that human behaviour can never be understood in terms of causes.

I have two complaints about the book. For its exorbitant price, one might reasonably have expected the publishers to have hired a more conscientious proof reader. The second complaint is more unusual: although the book raises interesting issues, it is too short to do justice to the complexity of its subject matter, and it should have been several times as long.

Stuart Sutherland

Stuart Sutherland is Director of the Centre for Research on Perception and Cognition at the University of Sussex, UK.

analyses presented are misleading. The section on the use of product inhibition as an aid to defining kinetic mechanism, for example, fails to consider the effects of abortive ternary complex formation in random mechanisms and thus predicts an inhibition pattern for the rapid-equilibrium case which is in fact rare. In addition failure to distinguish clearly between the exclusive and non-exclusive cases of the concerted model of co-operativity leads to confusion in the analysis of the curve shapes predicted by this system. The author's differentiation between the steady-state and rapid equilibrium treatments of kinetic mechanisms is also confused and this is particularly evident in his treatment of non-competitive inhibition.

The main strengths of this book lie in its treatments of the kinetics of coupled enzyme systems and of computer simulation methods. This latter chapter, which considers the use of both analogue and digital computers, is particularly valuable since this important topic is usually ignored in books of this type. The author's enthusiastic treatment should capture the interest of many students and although the section on digital methods would not be sufficient to enable the reader to write his own simulation program, it provides an excellent introduction to the terminology and methods used. The sections on the use of analogue computers define the components used and give sample programs for simple kinetic systems. These programs should be sufficient to stimulate many enzymologists with access to an analogue computer to take this approach further.

K. F. Tipton

K. F. Tipton is Lecturer in Biochemistry at the University of Cambridge, UK. 